\title{
A wide-range wavelength-tunable photon-pair source for characterizing single-photon detectors
}

\author{
LiJiong Shen, ${ }^{1}$ JianWei Lee, ${ }^{1}$ Antony Winata Hartanto, ${ }^{2}$ \\ PEngKian TAN, ${ }^{1}$ And Christian Kurtsiefer ${ }^{1,2,{ }^{*}}$ \\ ${ }^{1}$ Centre for Quantum Technologies, National University of Singapore, 3 Science Drive 2, Singapore 117543, \\ Singapore \\ ${ }^{2}$ Department of Physics, National University of Singapore, 2 Science Drive 3, Singapore 117551, Singapore \\ *christian.kurtsiefer@gmail.com
}

\begin{abstract}
The temporal response of single-photon detectors is usually obtained by measuring their impulse response to short-pulsed laser sources. In this work, we present an alternative approach using time-correlated photon pairs generated in spontaneous parametric down-conversion (SPDC). By measuring the cross-correlation between the detection times recorded with an unknown and a reference photodetector, the temporal response function of the unknown detector can be extracted. Changing the critical phase-matching conditions of the SPDC process provides a wavelength-tunable source of photon pairs. We demonstrate a continuous wavelength-tunability from $526 \mathrm{~nm}$ to $661 \mathrm{~nm}$ for one photon of the pair, and $1050 \mathrm{~nm}$ to $1760 \mathrm{~nm}$ for the other photon. The source allows, in principle, to access an even wider wavelength range by simply changing the pump laser of the SPDC-based source. As an initial demonstration, we characterize single photon avalance detectors sensitive to the two distinct wavelength bands, one based on Silicon, the other based on Indim Gallium Arsenide.
\end{abstract}

(C) 2020 Optical Society of America under the terms of the OSA Open Access Publishing Agreement

\section{Introduction}

Characterizing the temporal response function of single-photon detectors is crucial in timeresolved measurements, e.g. determining the lifetime of fluorescence markers [1], characterizing the spontaneous decay of single-photon emitters [2] and the photon statistics of astronomical sources [3] and measuring the joint spectral of photon-pair sources [4], so that the timing uncertainty contributed by the detection process can be taken into account. Typically, the temporal response of a detector is obtained from the arrival time distribution of photons collected from a pulsed laser. In this work, we present an alternative approach that leverages on the tight timing correlation [5] of photon pairs generated in spontaneous parametric down-conversion [6,7] (SPDC): the coincidence signature corresponding to the detection of two photons of the same pair is used to infer the temporal response function of the photodetectors. Compared to a pulsed laser, a SPDC source is easier to align, and is wavelength-tunable by changing the critical phase-matching condition of the SPDC process [8]. In addition, one can address two wavelength bands with the same source by choosing a non-degenerate phase matching condition.

For an initial demonstration, we generate photon pairs with a tunable wavelength range over $100 \mathrm{~nm}$ in the visible band, and over $700 \mathrm{~nm}$ in the telecommunication band - a tunability at least comparable to existing femtosecond pulsed lasers - and use it to characterize both Silicon (Si-APDs) and Indium Gallium Arsenide (InGaAs-APDs) avalanche photodiodes. In particular, we characterize timing the behaviour of a fast commercial Si-APD (Micro Photon Devices PD-050-CTC-FC) over a continuous wavelength range, for which we previously assumed an approximately uniform temporal response of the detector in the wavelength range from $570 \mathrm{~nm}$ to $810 \mathrm{~nm}$ [3]. With the measurement reported in this work, we observe a significant variation of 


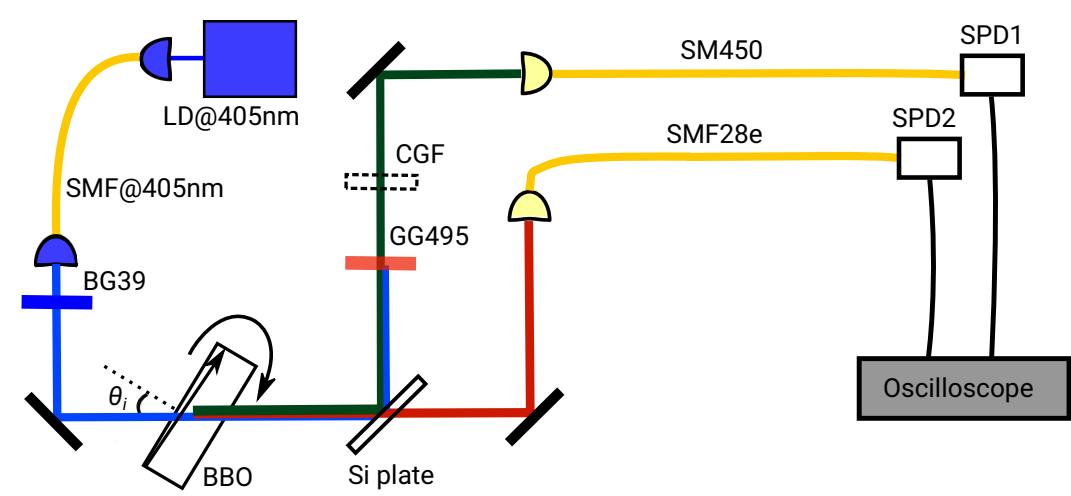

Fig. 1. Wavelength-tunable photon pair source based on Type-II SPDC. The critical phase-matching condition is changed by varying the angle of incidence $\theta_{i}$ of the pump at the crystal, in order to generate photon pairs at the desired wavelength in the visible and telecommunications band. A Silicon ( $\mathrm{Si}$ ) plate separates the photons in each pair. Tight timing correlations between photons in each pair, and a characterized detector SPD2, allow measuring the jitter of a single-photon detector (SPD1). A calibrated color glass filter (CGF) can be inserted to infer the wavelength of the photons sent to SPD1 using a transmission measurement. LD: laser diode, BBO: $\beta$-Barium Borate, SMF: single-mode fiber, GG495, BG39: color glass filters.

the timing jitter even on a relatively small wavelength interval of $\approx 10 \mathrm{~nm}$. A better knowledge of the timing response of this particular Si APD contributes to a better understanding of coherence properties of light in such experiments. Similarly, better characterization of the timing response over a wide wavelength range helps to better model fluorescence measurements regularly carried out with such detectors [1].

\section{Correlated photon pair source}

The basic configuration of the spontaneous parametric down conversion source is shown in Fig. 1. The output of a laser diode (central wavelength $\lambda_{p}=405 \mathrm{~nm}$, output power $10 \mathrm{~mW}$ ) is coupled to a single-mode optical fiber for spatial mode filtering, and focused to a Gaussian beam waist of $70 \mu \mathrm{m}$ into a $2 \mathrm{~mm}$ thick $\beta$-Barium Borate crystal as the nonlinear optical element, cut for Type-II phase matching $\left(\theta_{0}=43.6^{\circ}, \phi=30^{\circ}\right)$.

For this cut, SPDC generates photon pairs in the visible and telecommunications band, respectively. We collect the photons in a collinear geometry, with collection modes (beam waists $\approx 50 \mu \mathrm{m}$ ) defined by two single-mode fibers: one fiber (SMF450: single mode from $488 \mathrm{~nm}$ to $633 \mathrm{~nm}$ ) collects signal photons and delivers them to the single-photon detector SPD1, while the other fiber (standard SMF28e, single transverse mode from $1260 \mathrm{~nm}$ to $1625 \mathrm{~nm}$ ) collects idler photons and delivers them to SPD2. The signal and idler photons are separated to their respective fibers using a $100 \mu \mathrm{m}$-thick, polished Silicon ( $\mathrm{Si}$ ) plate as a dichroic element. The plate acts as a longpass filter (cut-off wavelength $\approx 1.05 \mu \mathrm{m}$ ), transmitting only the idler photons while reflecting approximately half of the signal photons.

To suppress uncorrelated visible and infrared photons detected by our SPDs, we insert both a blue color glass bandpass filter (BG39) in the pump path, attenuating parasitic emission from the pump laser diode and broadband fluorescence from the mode cleaning fiber, and a green color glass longpass filter (GG495) in the path of the idler photons to suppress pump light at SPD1. For the idler path, the silicon dichroic is sufficient. To tune the wavelength of down-converted photons, we change the critical phase-matching condition of the SPDC process by varying the angle of incidence $\theta_{i}$ of the pump beam at the crystal $[9,10]$. Figure 2 (red dots) shows the signal 


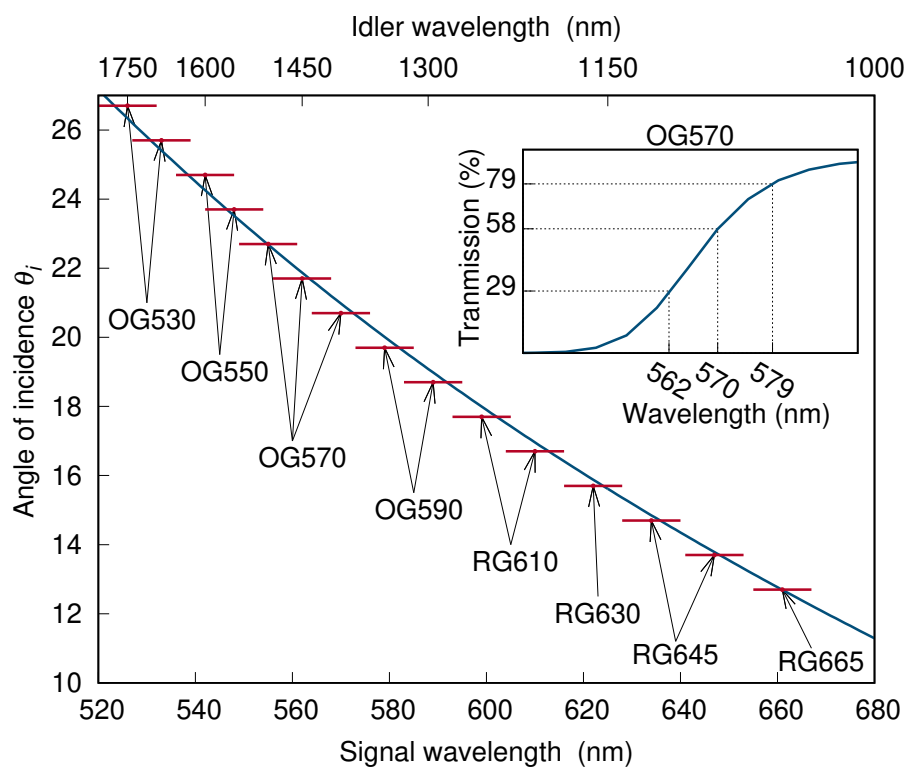

Fig. 2. Signal $\left(\lambda_{s}\right)$ and idler $\left(\lambda_{i}\right)$ wavelength dependence on the angle of incidence $\theta_{i}$ of the pump beam at the $\beta$-BBO crystal, produced in Type-II SPDC. We obtain $\lambda_{s}$ by measuring the transmission of signal photons through a set of calibrated color glass filters (OGs and RGs). $\lambda_{i}$ is calculated from $\lambda_{s}$ by the law of energy conservation. Error bars: uncertainty of the central transmission wavelength of the filters. Solid line: model predicting the output wavelengths based on energy and momentum conservation laws governing SPDC. Inset: the transmission-wavelength calibration curve of color glass filter OG570 used to experimentally determine three values of $\lambda_{s}$.

and idler wavelengths, $\lambda_{s}$ and $\lambda_{i}$, measured for our source for $\theta=12.7^{\circ}$ to $26.7^{\circ}$. To measure the signal wavelength $\lambda_{s}$, we insert different standardized color glass longpass filters (CGF in Fig. 1) for different angles $\theta_{i}$, and measure the transmission of the signal photons in order to infer their wavelength. The inset of Fig. 2 shows an example where a filter OG570 is used to infer $\lambda_{s}$ close to the cut-off wavelength of the filter. The corresponding idler wavelength is calculated through energy conservation in SPDC, $\lambda_{i}^{-1}=\lambda_{p}^{-1}-\lambda_{s}^{-1}$. Our measured SPDC wavelengths can be well described by a numerical phase matching model based on optical dispersion properties of BBO [11,12] (blue line).

This simple pair source provides photons in a wavelength range of $\lambda_{s}=526 \mathrm{~nm}$ to $661 \mathrm{~nm}$ and $\lambda_{i}=1050 \mathrm{~nm}$ to $1760 \mathrm{~nm}$, comparable with existing dye and solid-state femtosecond pulsed lasers $[13,14]$. In the following section, we demonstrate how the tight timing correlations of each photon pair can be utilized to characterize the temporal response of single photon detectors.

\section{Characterizing the temporal response of single-photon detectors}

The time response function $f(t)$ of a single photon detector characterizes the distribution of signal events at a time $t$ after a photon (of a sufficiently short duration) is absorbed by a detector. It characterizes the physical mechanism that converts a single excitation into a macroscopic signal, and can be measured e.g. recording the average response to attenuated optical pulses from a femtosecond laser [15]. In this paper, we use the timing correlation in a photon pair, which emerges at an unpredictable point in time. This requires two single photon detectors registering a photon. As the photon pair is correlated on a time scale of femtoseconds, and the relevant time scales for detector reponses is orders of magitudes larger, the correlation function $c_{12}(\Delta t)$ of 


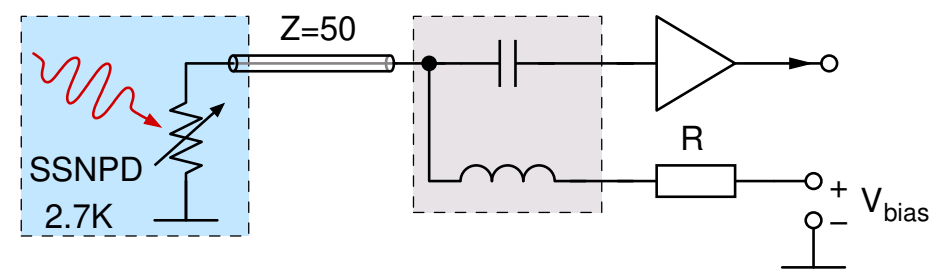

Fig. 3. Biasing and readout circuit for the superconducting nanowire single-photon detector (SNSPD). The SNSPD is current-biased using a constant voltage source and a series resistor $R$. When a photon is absorbed by the SNSPD, it changes temporarily from a superconducting to a conducting state. The resulting current change reaches a signal amplifier, which provides the photodetection signal.

time differences $\Delta t$ between the macroscopic photodetector signals is a convolution of the two detector response functions,

$$
c_{12}(\Delta t)=N\left(f_{1} * f_{2}\right)(\Delta t)=N \int f_{1}(t) f_{2}(\Delta t-t) d t,
$$

where $N$ the total number of recorded coincidence events. Obtaining the detector response function $f_{1}(t)$ from a measured correlation function $c_{12}(\Delta t)$ requires the known response function $f_{2}(t)$ of a reference detector. For a device under test, $f_{1}(t)$ can then be either reconstructed by fitting a $c_{12}(\Delta t)$ in Eqn. 1 with a reasonable model for $f_{1}(t ; P)$ (with a parameter set $P$ ) to a measured correlation function, or obtained from it via deconvolution.

To measure $c_{12}(\Delta t)$, we evaluate the detection time at single photon detector SPD1 by recording the analog detector signal with an oscilloscope, and interpolating the time it crosses a threshold of around half the average signal height with respect to a trigger event caused by a signal of single photon detector SPD2. The histogram of all time differences $\Delta t$ for many pair events then is a good represenation of $c_{12}(\Delta t)$.

\section{Reference detector characterization}

We use a superconducting nanowire detector (SNSPD) with a design wavelength at $1550 \mathrm{~nm}$ as the reference detector SPD2, because a SNSPD has an intrinsic wide-band sensitivity and fast temporal response. To determine its response function $f_{2}(t)$, we measure the correlation function $c_{12}$ from photon pairs with two detectors of the same model (Single Quantum SSPD-1550Ag).

Figure 3 shows the biasing and readout circuit of a single SNSPD. The SNSPD is kept at a temperature of $2.7 \mathrm{~K}$ in a cryostat, and is current-biased using a constant voltage source $\left(V_{\text {bias }}=1.75 \mathrm{~V}\right)$ and a series resistor $(R=100 \mathrm{k} \Omega)$ through a bias-tee at room temperature. The signal gets further amplified by $40 \mathrm{~dB}$ at room temperature to a peak amplitude of about $350 \mathrm{mV}$.

We first expose both detectors to photons at a wavelength of $810 \mathrm{~nm}$ using a degenerate PPKTP-based photon pair source pumped with a $405 \mathrm{~nm}$ laser diode (Fig. 4 (a)). The choice of using this source instead of the BBO-based source shown in Fig. 1 was borne out of convenience rather than from any limitation in our BBO-based source described before, as the PPKTP-based type-II SPDC source was readily available [16]. Figure 4 (b) shows the cross-correlation $c_{12}(\Delta t)$ for the two SNSPDs, normalized to background coincidences (red dots).

The histogram closely follows a Gaussian distribution (blue line) with standard deviation $\sigma_{12}=23.6(1) \mathrm{ps}$. This suggests that the two responses $f_{1}(t), f_{2}(t)$ are also Gaussian distributions, and Eqn. 1 can be simplified to

$$
c_{12}(\Delta t)=N G\left(\sigma_{12}, \Delta t\right)+C_{0}=N G\left(\sigma_{1}, \Delta t\right) * G\left(\sigma_{1}, \Delta t\right)+C_{0},
$$




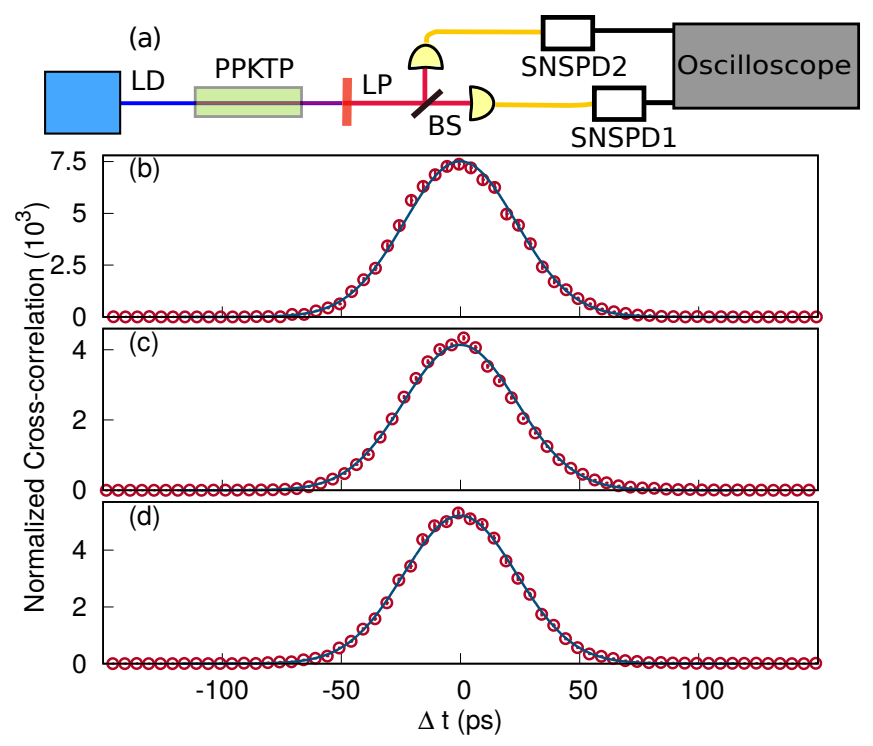

Fig. 4. (a) Simplified schematic of the PPKTP-based photon-pair source. We generate orthogonally-polarized, degenerate photon pairs at 810nm using Type-II SPDC, by pumping a PPKTP crystal $(9.55 \mu \mathrm{m}$ poling period) with a $405 \mathrm{~nm}$ laser beam. The photon pairs are separated by a polarizing beam-splitter and fibre-coupled to two SNSPDs. By changing the pump wavelength to $532 \mathrm{~nm}$, the same crystal generates $810 \mathrm{~nm}$ and $1550 \mathrm{~nm}$ photon pairs in a Type-0 SPDC process. We use a Si plate as a beam splitter to separate the non-degenerate photon pairs. BS: beam splitter, LD: laser diode, LP: longpass filter, PPKTP: Periodically Poled Potassium titanyl phosphate crystal. Cross-correlation of photodetection times registered by two SNSPDs detecting (b) degenerate $810 \mathrm{~nm}$ photon pairs, and (c) non-degenerate photon pairs at $810 \mathrm{~nm}$ and $1550 \mathrm{~nm}$ from the PPKTP-based source. (d) Cross-correlation of photodetection times of non-degenerate photon pairs at $548 \mathrm{~nm}$ and $1550 \mathrm{~nm}$ from the BBO-based source in Fig. 1.

where $N$ is the total number of correlated photon pairs detected, $G(\sigma, \Delta t)=e^{-\Delta t^{2} /\left(2 \sigma^{2}\right)} / \sqrt{2 \pi \sigma^{2}}$ is a normalized Gaussian distribution, and $C_{0}$ is associated with the accidental coincidence rate. The standard distribution of the correlation is then simply related to those of the individual detectors by $\sigma_{12}^{2}=\sigma_{1}^{2}+\sigma_{2}^{2}$. Assuming the same response for both detectors, we can infer at a wavelength of $810 \mathrm{~nm}$, corresponding to a the full-width at half-maximum (FWHM) of 39.2(2) ps.

Next, we calibrate the SNSPD at $1550 \mathrm{~nm}$ using photon pairs at $810 \mathrm{~nm}$ and $1550 \mathrm{~nm}$ generated from the same PPKTP-based SPDC source pumped with a $532 \mathrm{~nm}$ laser diode [Fig. 4 (a)]. The non-degenerate photon pairs are separated by a Si plate as a dicroic element. Figure 4 (c) shows the cross-correlation (red dots) of the photodetection times at the two SNSPDs, and a fit of a Gaussian distribution (blue line) with a standard deviation $\sigma_{12,810 / 1550}=23.8(2) \mathrm{ps}$. With $\sigma_{1,810}=16.7(1) \mathrm{ps}$ obtained at $810 \mathrm{~nm}$ previously, we obtain $\sigma_{2,1550}=\sqrt{\sigma_{12,810 / 1550}^{2}-\sigma_{1,810}^{2}}$ resulting in a timing jitter of 39.9(6) ps (FWHM) of the SNSPD at $1550 \mathrm{~nm}$.

Finally, to determine the temporal response function of a SNSPD at $548 \mathrm{~nm}$, we used the BBO-based pair source [Fig. 1] to prepare non-degenerate photon pairs at $548 \mathrm{~nm}$ and $1550 \mathrm{~nm}$. Figure 4 (d) shows the cross-correlation obtained with our detectors. The fit to a Gaussian distribution (blue line) leads to a standard deviation $\sigma_{12,548 / 1500}=23.7(1) \mathrm{ps}$. With the same argument as before, and using $\sigma_{1,1500}=16.9(2) \mathrm{ps}$, we obtain a timing jitter of 38.9(7) ps (FWHM) at $548 \mathrm{~nm}$. So in summary, the timing jitter of the SNSPD shows no statistically 


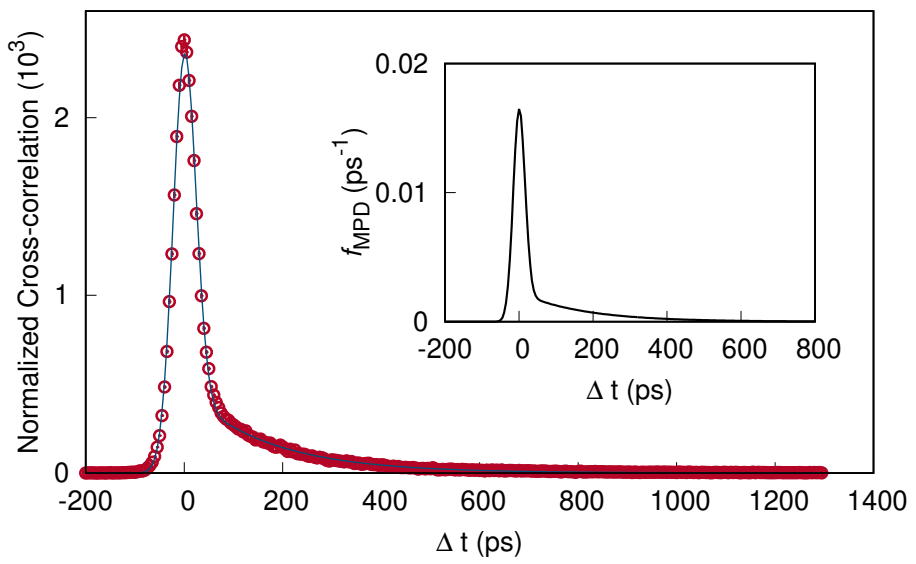

Fig. 5. Cross-correlation between photodetection times at a Si-APD and a characterized SNSPD, normalized to background coincidences $g^{(2)}(\Delta t)$. The detectors were illuminated by a non-degenerate $(555 \mathrm{~nm}, 1500 \mathrm{~nm})$ photon pair source. By fitting the data (red dots) to a model (blue line) obtained by convolving the individual temporal response model of both detectors, we are able to extract parameters describing the temporal response of the Si-APD (inset). $\Delta t$ : photodetection time difference.

significant dependency on the wavelength in our measurements.

The timing jitter partually originates from the threshold detection mechanism: for a photodetection signal $V(t)$, the timing uncertainty $\sigma_{t}$ for crossing a threshold, contributed by the electrical noise $\sigma_{V}$, is given by $\sigma_{t \text {;noise }}=\sigma_{V} /(d V / d t)$ at the threshold [17, 18]. For our SNSPDs, we estimate $\sigma_{t \text {;noise }} \approx 15 \mathrm{ps}$, corresponding to a contribution of about $35 \mathrm{ps}$ to the timing jitter of the combined SNSPD and electronic readout system, i.e., we are dominated by this electrical noise. The jitter of the oscilloscope is claimed to be a few ps, which suggests that the intrinsic jitter of these SNSPDs is about $10-20$ ps (FWHM) [19].

In the following section, we use the standard deviation $\sigma_{2}$ obtained at these wavelengths to define the temporal response function of the reference detector $f_{2}=G\left(\sigma_{2}\right)$ in Eqn. 1, and use the method outlined in Sec. 3 to characterize $f_{1}$ of an unknown detector.

\section{Avalanche photodetector characterization}

First, we characterize the temporal response function $f_{\mathrm{Si}}$ of a thin Silicon avalanche photodiode (Si-APD) from Micro Photon Devices (PD-050-CTC-FC). Although thin Si-APDs have been characterized in previous works at a few discrete wavelengths [20-22], there has yet been a characterization performed over a continuous wavelength range.

Following Refs. [3,23], we describe the temporal response function with a heuristic model

$$
f_{\mathrm{Si}}(\Delta t)=A G(\sigma, \Delta t-\mu)+B G(\sigma, \Delta t-\mu) * e^{-(\Delta t-\mu) / \tau},
$$

a combination of a Gaussian component of mean $\mu$ and standard deviation $\sigma$, and an exponential term with a characteristic decay constant $\tau$. The weights of each distribution are described by $A$ and $B$. The Gaussian component is associated with an avalanche that occurs due to the absorption of a photon in the depletion region. The exponential component, convoluted with a Gaussian distribution, is associated with an avalanche that is initiated by a photoelectron that diffused into the depletion region produced by photon absorption elsewhere.

We characterize the Si-APD over a wavelength range from $\lambda_{1}=542 \mathrm{~nm}$ to $647 \mathrm{~nm}$ in steps of about $10 \mathrm{~nm}$. The photon wavelength is tuned by rotating the crystal, changing the angle 


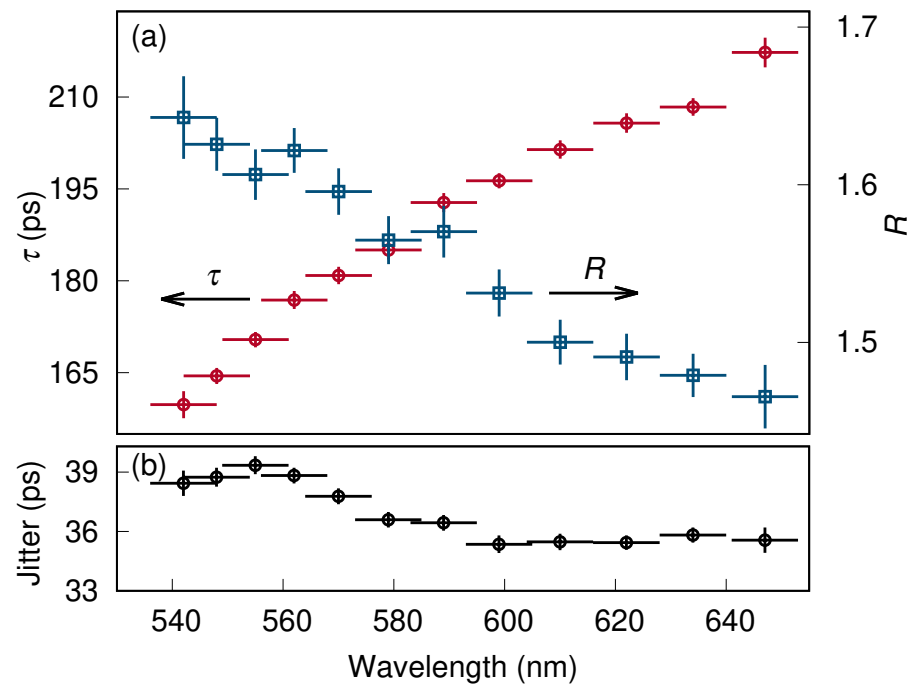

Fig. 6. (a) Si-APD temporal response characteristics: ratio $R$ between the Gaussian and the exponential component of the temporal response function (red), and characteristic decay constant $\tau$ of the exponential component(blue). (b) Measured timing jitter of a $\mathrm{Si}$-APD as a function of wavelength. Horizontal error bars: uncertainty of the cut-off wavelength of the color glass filter used to measure photon wavelength. Vertical error bars: fit error of $R, \tau$, and timing jitter.

of incidence $\theta_{i}$ of the pump from $13.7^{\circ}$ to $24.7^{\circ}$, in steps of $1^{\circ}$. For each $\theta_{i}$, we obtain the cross-correlation $c_{12}(\Delta t)$ similarly as in section 4 . Figure 5 (red dots) shows $g^{(2)}$, the crosscorrelation normalized to background coincidences, obtained when signal and idler wavelengths are $\lambda_{1}=555 \mathrm{~nm}$ and $\lambda_{2}=1500 \mathrm{~nm}$, respectively. For every $\left(\theta_{i}, \lambda_{1}, \lambda_{2}\right)$, we deduce $f_{\mathrm{Si}}$ by fitting the measured $c_{12}$ to the model in Eqn. 1 with $f_{1}=f_{\mathrm{Si}}$, and $f_{2}$ a Gaussian distribution with full-width at half-maximum (39.9 ps) corresponding to the SNSPD jitter at $1550 \mathrm{~nm}$. For the SNSPD, we assume that its jitter remains constant over the wavelength range $\lambda_{2}=1082 \mathrm{~nm}$ to $1602 \mathrm{~nm}$, motivated by the observation that it does not differ significantly for $\lambda_{2}=810 \mathrm{~nm}$ and $1550 \mathrm{~nm}$. The fit results in parameters $\sigma$ and $\tau$ which characterize $f_{\mathrm{Si}}$ at the corresponding wavelength $\lambda_{1}$. Figure 5 (inset) shows $f_{\mathrm{Si}}(\Delta t)$ for $\lambda_{1}=555 \mathrm{~nm}$.

Two figures of merit are of interest for characterizing the thin Si APD: the duration $\tau$ of the exponential tail, and the ratio $R$ between the coincidences attributed to the Gaussian component to those attributed to the exponential component,

$$
R=\frac{\int_{-\infty}^{\infty} A G(\sigma, \Delta t-\mu)}{\int_{-\infty}^{\infty} B G(\sigma, \Delta t-\mu) * e^{-(\Delta t-\mu) / \tau}}=\frac{A}{B \tau} .
$$

Both values determine if the full-width at half-maximum (FWHM), a value typically quoted for the detector jitter, serves as a good figure of merit for the temporal response of a detector. For example, the jitter of a detector with $R \ll 0.5$ and $\sigma \ll \tau$, is better described by $\tau$ than the FWHM of the temporal response function.

Figure 6 (a) shows that $R$ reduces while $\tau$ increases with increasing wavelength. The detector jitter (FWHM) is shown in Figure 6(b). The observation that $\tau$ changes significantly with wavelength is especially revelant for fluorescence lifetime measurements, where the exponential tail in the temporal response function can be easily misattributed to fluorescence when the 


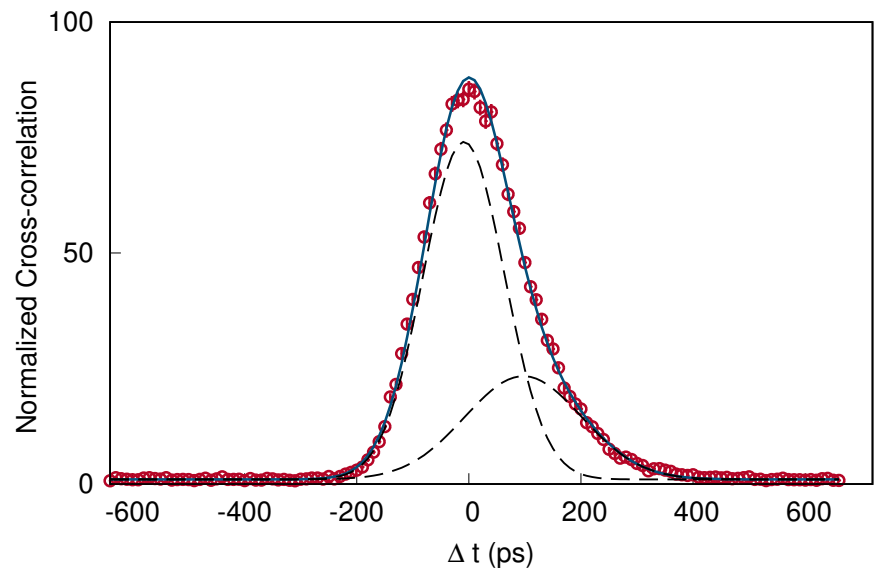

Fig. 7. Cross-correlation function normalized to background coincidences $g^{(2)}(\Delta t)$ of the InGaAs-APD and the reference SNSPD. The cross-correlation approximates the InGaAs-APD temporal response well since the latter is much slower than the SNSPD. We fit the measured $g^{(2)}(\Delta t)$ (red dots) with a model consisting of two Gaussian distributions (solid line) with an overall width of 196 ps (FWHM). Dashed lines: individual Gaussian components, $\Delta t$ : time difference between the photodetection times.

detector is not characterized at the wavelength of interest [1].

Next, we characterize the temporal response function $f_{\text {InGaAs }}$ of an InGaAs avalanche photodiode (S-Fifteen Instruments ISPD1) which is sensitive in the telecommunication band. We extract $f_{\text {InGaAs }}$ by measuring the cross-correlation $c_{12}$ of the detection times between the InGaAs-APD and our reference SNSPD. We note that since the expected jitter of the InGaAs-APD $(\approx 200 \mathrm{ps})$ is significantly larger than that of the $\operatorname{SNSPD}(\approx 40 \mathrm{ps}), f_{\text {InGaAs }}$ is well-approximated by $c_{12}$.

Again, we fit $c_{12}(\Delta t)$ to a heuristic model [24], here comprising of a linear combination of two Gaussian distributions

$$
c_{12}(\Delta t) \approx N f_{\text {InGaAs }}(\Delta t)+C_{0}=N\left[A G\left(\mu_{1}, \sigma_{1}, \Delta t\right)+B G\left(\mu_{2}, \sigma_{2}, \Delta t\right)\right]+C_{0},
$$

where $A$ and $B$ are the weights of each distribution, $\mu_{1}\left(\mu_{2}\right)$ and $\sigma_{1}\left(\sigma_{2}\right)$ is the mean and standard deviation characterizing the Gaussion distribution $\mathrm{G}$, and $C_{0}$ is associated with the accidental coincidence rate. Figure 7 shows the measured cross-correlation $c_{12}$ (red dots) and the fit result (blue line) when the InGaAs-APD detected photons with a wavelength of $1200 \mathrm{~nm}$.

We tune the wavelength of the photons sent to the InGaAs-APD from $1200 \mathrm{~nm}$ to $1600 \mathrm{~nm}$ in steps of $100 \mathrm{~nm}$, and obtain $c_{12}$ for each wavelength. Figure 8 shows the parameters describing the temporal response of the InGaAs-APD: its jitter, the ratio $R=A / B$ of the two Gaussian distributions contributing to $f_{\text {InGaAs }}$, the temporal separation between the two Gaussian distributions $\left(\mu_{1}-\mu_{2}\right)$, and the standard deviation of the two Gaussian distributions $\left(\sigma_{1}, \sigma_{2}\right)$. We find no significant variation of any parameter over the entire wavelength range.

\section{Conclusion}

We have presented a widely-tunable, non-degenerate photon-pair source that produces signal photons in the visible band, and idler photons in the telecommunications band. With the source, we demonstrate how the tight-timing correlations within each photon pair can be utilized to characterize single-photon detectors. This is achieved by measuring the cross-correlation of the detection times registered by the device-under-test (DUT), and a reference detector - an SNSPD, which has a relatively low and constant jitter over the wavelength range of interest. By 

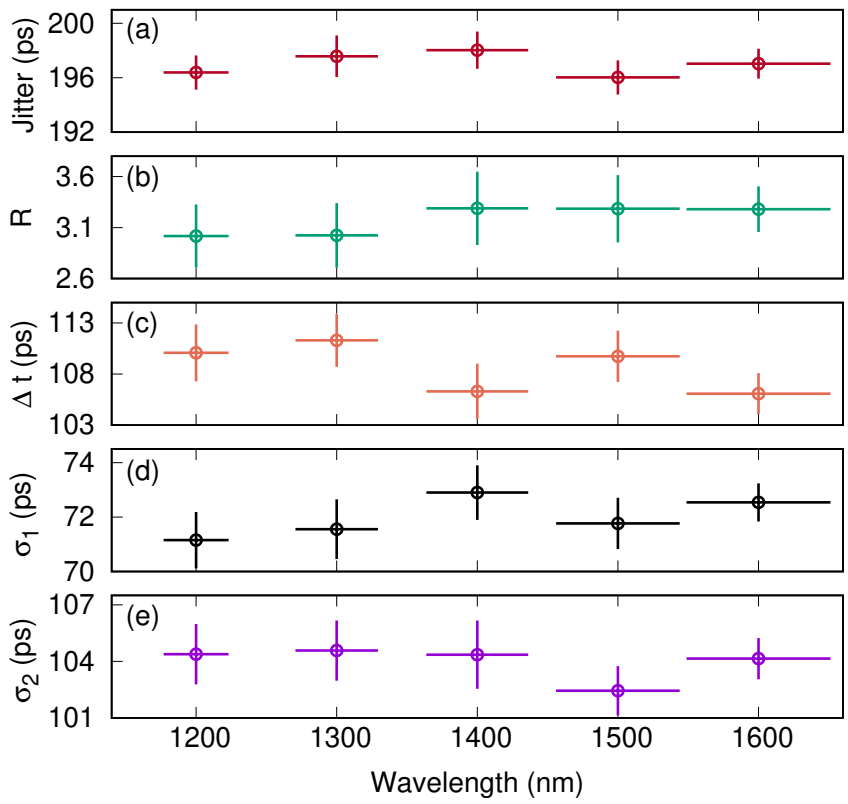

Fig. 8. Parameters describing the temporal response function $f_{\text {InGaAs }}$ of an InGaAsAPD, measured over a wide wavelength range for a linear combination of two Gaussian distributions to model $f_{\text {InGaAs }}$ (Eqn. 5). The parameters are extracted by fitting the measured temporal response to this model: (a) timing jitter, (b) the weight ratio $R$ of the Gaussian distributions, (c) the temporal separation $\mu_{1}-\mu_{2}$ between the Gaussian distributions, (d) and (e) the standard deviations $\sigma_{1}$ and $\sigma_{2}$ of the Gaussian distributions.

taking into account the jitter introduced by the reference detector, we are able to extract the temporal response function of the DUT. As the source is based on SPDC in a BBO crystal, its output wavelengths are continuously tunable by varying the angle of incidence of the pump at the crystal. We experimentally demonstrated wavelength-tunability of over $100 \mathrm{~nm}$ in the visible band, and over $700 \mathrm{~nm}$ in the telecommunications band - a similar tunability compared to existing femtosecond pulsed laser systems.

With our source, we measured the temporal response functions of two single-photon detectors, an Si-APD and an InGaAs-APD, over a continuous wavelength range centered at the visible and telecommunications band, respectively. For the InGaAs-APD, we observed no significant variation of its jitter over a wide wavelength range. For the Si-APD, we observed that the exponential component of its temporal response increases with wavelength. This observation emphasizes the need for an accurate accounting of Si-APD jitter in precision measurements, e.g. characterizing fluorescence markers at the wavelength of interest [1], or measuring the photon statistics of narrowband astronomical sources [3].

\section{Funding}

This research was supported by the National Research Foundation (NRF) Singapore (Grant: QEP-P1), and through the Research Centres of Excellence programme supported by NRF Singapore and the Ministry of Education, Singapore. 


\section{References}

1. W. Becker, Advanced time-correlated single photon counting techniques, vol. 81 (Springer Science \& Business Media, 2005).

2. M. J. Stevens, R. H. Hadfield, R. E. Schwall, S. W. Nam, R. P. Mirin, and J. A. Gupta, "Fast lifetime measurements of infrared emitters using a low-jitter superconducting single-photon detector,” Appl. Phys. Lett. 89, 031109 (2006).

3. P. K. Tan, A. H. Chan, and C. Kurtsiefer, "Optical intensity interferometry through atmospheric turbulence," Mon. Notices Royal Astron. Soc. 457, 4291-4295 (2016).

4. K. Zielnicki, K. Garay-Palmett, D. Cruz-Delgado, H. Cruz-Ramirez, M. F. O’Boyle, B. Fang, V. O. Lorenz, A. B. U'Ren, and P. G. Kwiat, "Joint spectral characterization of photon-pair sources,” J. Mod. Opt. 65, 1141-1160 (2018).

5. C.-K. Hong, Z.-Y. Ou, and L. Mandel, "Measurement of subpicosecond time intervals between two photons by interference," Phys. review letters 59, 2044 (1987).

6. D. N. Klyshko, A. N. Penin, and B. F. Polkovnikov, "Parametric luminescence and light scattering by polaritons," JETP Lett. 11, 5-8 (1970).

7. D. C. Burnham and D. L. Weinberg, "Observation of simultaneity in parametric production of optical photon pairs," Phys. Rev. Lett. 25, 84-87 (1970).

8. S. E. Harris, "Tunable optical parametric oscillators," Proc. IEEE 57, 2096-2113 (1969).

9. R. L. Sutherland, Handbook of nonlinear optics (CRC press, 2003), 2nd ed.

10. R. W. Boyd, Nonlinear Optics (Academic Press, Inc., USA, 2008), 3rd ed.

11. D. Eimerl, L. Davis, S. Velsko, E. K. Graham, and A. Zalkin, "Optical, mechanical, and thermal properties of barium borate," J. Appl. Phys. 62, 1968-1983 (1987).

12. D. N. Nikogosyan, "Beta barium borate (BBO) - A review of its properties and applications," Appl. Phys. A Solids Surfaces 52, 359-368 (1991).

13. F. J. Duarte, P. Kelley, L. W. Hillman, and P. F. Liao, Dye laser principles: with applications (Academic Press, 1990).

14. E. Sorokin, S. Naumov, and I. T. Sorokina, "Ultrabroadband infrared solid-state lasers," IEEE J. Sel. Top. Quantum Electron. 11, 690-712 (2005).

15. A. Lamas-Linares and C. Kurtsiefer, "Breaking a quantum key distribution system through a timing side channel," Opt. Express 15, 9388-9393 (2007).

16. L. Shen, J. Lee, L. P. Thinh, J.-D. Bancal, A. Cerè, A. Lamas-Linares, A. Lita, T. Gerrits, S. W. Nam, V. Scarani, and C. Kurtsiefer, "Randomness extraction from bell violation with continuous parametric down-conversion," Phys. Rev. Lett. 121, 150402 (2018).

17. G. Bertolini and A. Coche, Semiconductor Detectors (North-Holland, 1968).

18. J. Wu, L. You, S. Chen, H. Li, Y. He, C. Lv, Z. Wang, and X. Xie, "Improving the timing jitter of a superconducting nanowire single-photon detection system," Appl. Opt. 56, 2195-2200 (2019).

19. B. Korzh, Q.-Y. Zhao, J. P. Allmaras, S. Frasca, T. M. Autry, E. A. Bersin, A. D. Beyer, R. M. Briggs, B. Bumble, M. G. M. C. Colangelo, A. E. Dane, T. Gerrits, F. Marsili, G. Moody, E. Ramirez, J. D. Rezac, M. J. Stevens, E. E. Wollman, D. Zhu, P. D. Hale, K. L. Silverman, R. P. Mirin, S. W. Nam, M. D. Shaw, and K. K. Berggren, "Demonstration of sub-3 ps temporal resolution with a superconducting nanowire single-photon detector," Nat. Photonics 14, 250-255 (2020).

20. A. Lacaita, M. Ghioni, and S. Cova, "Double epitaxy improves single-photon avalanche diode performance,” Electron. Lett. 25, 841-843 (1989).

21. A. Giudice, M. Ghioni, R. Biasi, F. Zappa, S. Cova, P. Maccagnani, and A. Gulinatti, "High-rate photon counting and picosecond timing with silicon-spad based compact detector modules," J. Mod. Opt. 54, 225-237 (2007).

22. S. Cova, G. Ripamonti, and A. Lacaita, "Avalanche semiconductor detector for single optical photons with a time resolution of 60 ps,” Nucl. Instruments Methods Phys. Res. Sect. A: Accel. Spectrometers, Detect. Assoc. Equip. 253, 482 - 487 (1987).

23. Q. Hernandez, D. Gutierrez, and A. Jarabo, “A Computational Model of a Single-Photon Avalanche Diode Sensor for Transient Imaging,” arXiv:1703.02635 (2017).

24. A. Tosi, F. Acerbi, A. Dalla Mora, M. A. Itzler, and X. Jiang, "Active area uniformity of ingaas/inp single-photon avalanche diodes," IEEE Photonics J. 3, 31-41 (2011). 